\title{
Circularly Polarized Luminescence from Axially Chiral BODIPY DYEmers: an Experimental and Computational Study
}

\author{
Francesco Zinna, ${ }^{[a]}$ Torsten Bruhn, ${ }^{[b]}$ Ciro A. Guido, ${ }^{[a]}$ Johannes Ahrens, ${ }^{[c]},+$ Martin Bröring, ${ }^{[c]}$ Lorenzo Di \\ Bari, ${ }^{[a] *}$ Gennaro Pescitelli[a]*
}

The paper is dedicated to the memory of our colleague and friend Ettore Castiglioni

\begin{abstract}
With our new home-built CPL instrument, we measured fluorescence and CPL spectra of the enantiomeric pairs of two quasi-isomeric BODIPY DYEmers 1 and 2, endowed with axial chirality. The ECD and CPL spectra of these atropisomeric dimers are dominated by the exciton coupling between the main $\pi-\pi^{*}$ transitions (550-560 nm) of the two BODIPY rings. Compound 1 has strong ECD and CPL spectra $\left(g_{\text {lum }}=4 \cdot 10^{-3}\right)$ well reproduced by TDDFT and SCS-CC2 calculations using DFT-optimized groundand excited-state structures. Compound 2 has weaker ECD and CPL spectra $\left(g_{\mathrm{lum}}=4 \cdot 10^{-4}\right)$, partly due to the mutual cancellation of electric-electric and electric-magnetic exciton couplings, and partly to its conformational freedom. This compound is computationally very challenging. Starting from the optimized excited-state geometries, we predicted the wrong sign for the CPL band of 2 using TDDFT with the most recommended hybrid and range-separated functionals, whereas only SCS-CC2 or a DFT functional with full exact exchange percentage provided the correct sign.
\end{abstract}

\section{Introduction}

Chiral non-racemic compounds can emit left and right circularly polarized light with different intensities. This phenomenon can occur in photoluminescence, chemiluminescence and electroluminescence, and it is called circularly polarized luminescence $(\mathrm{CPL})$. $^{[1-2]}$

CPL may find applications in several technological fields, such as chiral optoelectronics and photonics (in devices able to emit or detect CPL), ${ }^{[3-6]}$ spintronics, ${ }^{[7-8]}$ information technology (e.g. in optical data storage) ${ }_{,}^{[9]}$ and photoswitches. ${ }^{[10]}$

The extent of CPL is usually quantified by means of the luminescence dissymmetry factor $g_{\mathrm{lum}} \cdot{ }^{[1-2]}$

$$
g_{\text {lum }}=\frac{\mathrm{I}_{\mathrm{L}}-\mathrm{I}_{\mathrm{R}}}{\frac{1}{2}\left(\mathrm{I}_{\mathrm{L}}+\mathrm{I}_{\mathrm{R}}\right)}=\frac{\Delta \mathrm{I}}{\mathrm{I}}
$$

where $I_{L}$ and $I_{R}$ are the left and right polarized components of the

[a] F. Zinna, Dr C. A. Guido, Prof. L. Di Bari, Prof. G. Pescitell Dipartimento di Chimica e Chimica Industriale, Università di Pisa, via Moruzzi 13, 56124 Pisa, Italy. e-mail lorenzo.dibari@unipi.it, gennaro.pescitelli@unipi.it

[b] Dr T. Bruhn, Institut für Organische Chemie, Universität Würzburg Am Hubland, 97074 Würzburg, Germany.

[c] Dr J. Ahrens, Prof. M. Bröring, Institut für Anorganische und Analytische Chemie, Technische Universität Braunschweig, Hagenring 30, 38106 Braunschweig, Germany.

$\dagger \quad$ Present address: Department of Organic Chemistry, Weizmann Institute of Science, 76100 Rehovot, Israel.

Supporting information for this article is available on the WWW under http://dx.doi.org/10.1002/chem.2016xxxxx. . emission, respectively. For non-aggregated organic molecules, common $g_{\text {lum }}$ values hardly go over $10^{-3},{ }^{[11-12]}$ while it is possible to reach considerably higher values $(0.1-1)$ for certain selected bands of chiral lanthanide complexes. ${ }^{[13-15]}$ In order to have small chiral organic molecules suitable for practical applications, such as circularly polarized organic light emitting diodes (CPOLED), ${ }^{[16]}$ the design of chiral systems endowed with a high $g_{\text {lum }}$ is desirable. To accomplish this task, quantum mechanical calculations can guide the rational design of efficient CP fluorophores by means of the insight in the chirality of the excited state.

Boron dipyrrin derivatives (BODIPY, 4,4-difluoro-4-bora-3a,4adiaza-s-indacene) are widely studied fluorophores due to their high absorption coefficients and high quantum yields which result in high brightness. ${ }^{[17-18]}$ Moreover it is possible to finely tune their spectroscopic properties modifying the substituents on the pyrrole rings or on the boron atom. These features make them suitable to be employed in OLEDs or as light-emitting probes and bio-probes, and prompted some research groups to develop chiral BODIPYs (or analogues) capable of emitting circularly polarized light. ${ }^{[19-26]}$ In the present work, we studied the circularly polarized emission properties of BODIPY "DYEmers" 1 and 2 (Scheme 1). In the two enantiomeric pairs of atropisomeric dimers, the chirality is brought about by the chirality axis coincident with the bond connecting the two BODIPY monomers. This aspect strongly differentiates our system from the design of previously reported chiral BODIPYs. ${ }^{[19-26]}$ Compounds 1 and 2 are quasi-isomers differing in the position of the aryl-aryl junction, namely $\mathrm{C} 3 / \mathrm{C}^{\prime}$ ' (ring a atoms) in 1, and C1/C1' (ring $\beta^{\prime}$ atoms) in 2 (Scheme 1). Perfect isomerism is broken by the replacement of two methyl groups in $\mathbf{1}$ by ethyl ones in $\mathbf{2}$.
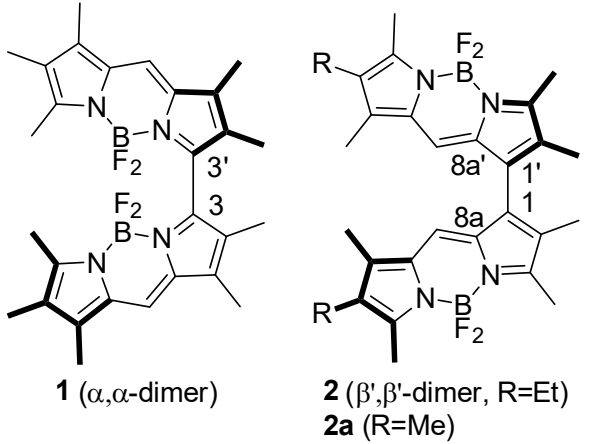

Scheme 1. Structures of quasi-isomeric BODIPY DYEmers 1 and 2, and of model $2 \mathbf{a}$.

In previous communications, we reported the synthesis, enantioseparation, and absolute stereochemistry assignment of 
1 and 2. ${ }^{[27]}\{$ Nepomnyashchii, 2011 \#67\} These compounds display clear-cut exciton ECD couplets ${ }^{[28]}$ in the long-wavelength region between 400 and $650 \mathrm{~nm}$, allied with the first $\pi-\pi^{*}$ transition of the BODIPY chromophore which is long-axis polarized (ECD spectra are reproduced in Figures 1 and 2 below). For both compounds the details of the exciton coupling are very interesting. Compound $\mathbf{1}$ obeys the exciton chirality rule therefore the (aR)-enantiomer, endowed with $\mathrm{M}$ chirality, shows a strong negative exciton couplet with an exceptionally large band splitting of $73 \mathrm{~nm}(0.33 \mathrm{eV})$. On the contrary, compound 2 violates the exciton coupling rule, and the (aR)-enantiomer, endowed with $M$ chirality, shows a weak positive exciton couplet. The reason for such an apparent exception was found in the strong intrinsic transition magnetic dipole moments $m$ allied with the discussed transition. The electric/magnetic or $\mu-m$ coupling has the same sign as the electric-electric $\mu-\mu$ coupling for isomer 1. On the contrary, the two couplings have opposite signs for isomer 2 , the $\mu-m$ one overwhelms the $\mu-\mu$ one, and the couplet sign is reversed. ${ }^{[27,29]}$ Such a situation is not unique to BODIPY DYEmers but still very infrequent. ${ }^{[29]}$

While the ground state chiroptical properties of compound 1 and 2 were fully investigated before, ${ }^{[27,29]}$ a characterization of their excited states has not been reported yet. We decided to take advantage of our newly rebuilt spectrofluoropolarimeter (see description in the Supporting Information) to carry out the CPL measurements of $\mathbf{1}$ and $\mathbf{2}$. The experimental results were corroborated by a detailed computational investigation. In particular, it was interesting to study the CPL behaviour of compounds showing the peculiar exciton-coupled ECD spectra discussed above. In fact, bis-chromophoric exciton-coupled compounds have been studied systematically only in the related context of fluorescence detected CD (FDCD), ${ }^{[30]}$ while covalent exciton-coupled systems have been considered only occasionally in CPL measurements, ${ }^{[31-36]}$ On the contrary, there is much interest in CPL as a means for the detection and characterization of chiral supramolecular aggregates of chromophoric species, which are of course also exciton-coupled. In this context, CPL has demonstrated exceptional sensitivity to long-range couplings and has therefore been used as a probe of supramolecular structure, mode of aggregation, degree of disorder, and other properties. ${ }^{[31,34,37-39]}$ \{Hall, 2016 \#90; Hall, $2016 \# 89\}$

\section{Results and Discussion}

\section{Experimental emission and CPL spectra}

Both compounds $\mathbf{1}$ and $\mathbf{2}$ are highly luminescent and therefore good quality emission and CPL spectra could be obtained. Our instrument, described in the Supporting Information, is capable to measure $\mathrm{CPL}$ and total florescence simultaneously. Emission and CPL spectra of $\mathbf{1}$ and $\mathbf{2}$ are shown in Figure 1, and the relevant numerical data are summarized in Table 1. Absorption and ECD spectra of 1 and $\mathbf{2}$ have been reported before. ${ }^{[27]}$ They were re-measured and, together with the respective data, are reproduced in Figure 1 and Table 1 for a direct comparison with their emissive counterparts.

Compound 1 displayed a relatively strong CPL signal with a high signal-to-noise $(\mathrm{S} / \mathrm{N})$ ratio. The fluorescence band is Gaussianshaped with a maximum around $655 \mathrm{~nm}$, and it is retraced by the CPL spectra. A very large Stokes shift is measured, amounting to $95 \mathrm{~nm}(0.32 \mathrm{eV})$, confirming previous measurements on the same compound. ${ }^{[0]}$ The Stokes shifts normally observed for monomeric unsubstituted BODIPY dyes are much smaller $(10-15 \mathrm{~nm})$. Our value surpasses those observed for purposely designed energy-transfer cassettes ${ }^{[17-18]}$ and reaches those of similar BODIPY dimers. ${ }^{[41-42]}$ The CPL spectra for the two enantiomers of $\mathbf{1}$ are mirror images, as expected (positive for (aS)-1 and negative for $(a R)-1)$, with a $g_{\text {lum }}= \pm 3.8 \cdot 10^{-3}$. This value is similar to that reported for other chiral BODIPY derivatives, ${ }^{[19-24]}$ and on the upper edge of the range reported for chiral non aggregated small organic molecules. ${ }^{[11]}$ Larger $g_{\text {lum }}$ values may however be obtained with BODIPY-based polymers. ${ }^{[43]}$

The comparison of the CPL with the ECD spectra shows that the most red-shifted Cotton effect (at $560 \mathrm{~nm}$ ) has the same sign of the CPL spectrum (Figure 1). This appears to be a general behavior for ECD/CPL spectra of organic compounds, including all exciton-coupled systems investigated to date, ${ }^{[31-32,34-36]}$ at least in cases where no substantial geometrical rearrangement occurs between the ground and excited state. ${ }^{[44]}$

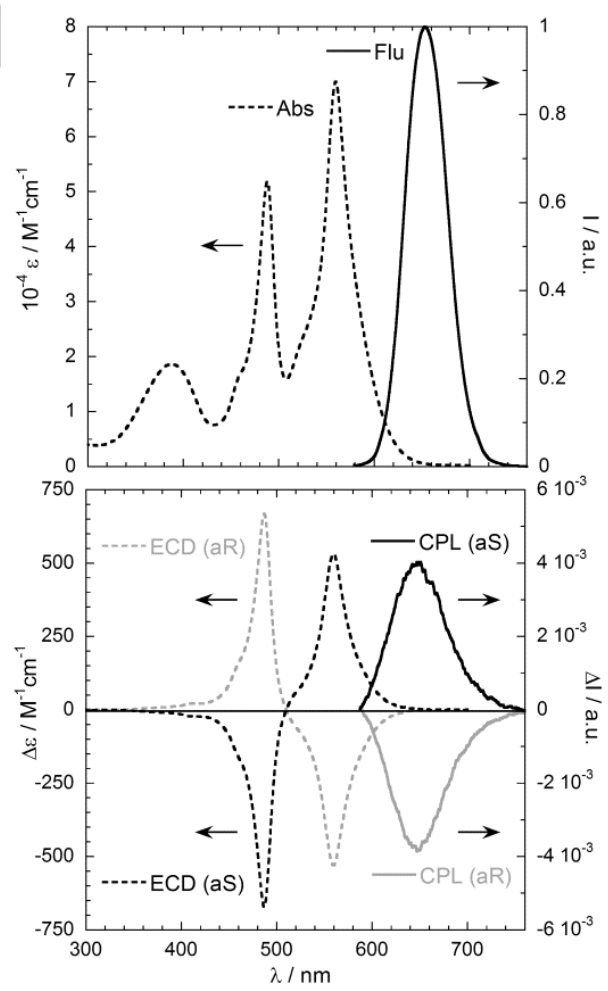

Figure 1. Experimental absorption and fluorescence spectra (top), ECD and CPL spectra (bottom) of compound 1. The scale of the CPL is normalized to the maximum of emission, so that the glum values can be directly read on $y$ axis at the maximum of each curve. 
Table 1. Spectroscopic experimental and computational data for compounds 1 and 2

\begin{tabular}{|c|c|c|c|c|c|c|c|c|}
\hline \multicolumn{9}{|c|}{ Compound 1 ( $\alpha, \alpha$ dimer) } \\
\hline \multicolumn{3}{|c|}{ Experimental $^{[\mathrm{a}]}$} & \multicolumn{3}{|c|}{ Calculated TDDFT ${ }^{[b]}$} & \multicolumn{3}{|c|}{ Calculated $\mathrm{CC} 2^{[\mathrm{c}]}$} \\
\hline Absorption & Emission & Stokes shift & Absorption & Emission & Stokes shift & Absorption & Emission & Stokes shift \\
\hline $\begin{array}{c}560 \mathrm{~nm} \\
(2.21 \mathrm{eV})\end{array}$ & $\begin{array}{c}655 \mathrm{~nm} \\
(1.89 \mathrm{eV})\end{array}$ & \multirow{3}{*}{$\begin{array}{c}95 \mathrm{~nm} \\
(0.32 \mathrm{eV})\end{array}$} & $\begin{array}{c}471 \mathrm{~nm} \\
(2.63 \mathrm{eV})\end{array}$ & $\begin{array}{c}545 \mathrm{~nm} \\
(2.28 \mathrm{eV})\end{array}$ & \multirow{3}{*}{$\begin{array}{c}74 \mathrm{~nm} \\
(0.35 \mathrm{eV})\end{array}$} & $\begin{array}{c}480 \mathrm{~nm} \\
(2.58 \mathrm{eV})\end{array}$ & $\begin{array}{c}539 \mathrm{~nm} \\
(2.30 \mathrm{eV})\end{array}$ & \multirow{3}{*}{$\begin{array}{c}59 \mathrm{~nm} \\
(0.28 \mathrm{eV})\end{array}$} \\
\hline$g_{\text {abs }}$ & $g_{\text {lum }}$ & & $g_{\mathrm{abs}}$ & $g_{\text {lum }}$ & & $g_{\text {abs }}$ & $g_{\text {lum }}$ & \\
\hline $9.5 \cdot 10^{-3}$ & $3.8 \cdot 10^{-3}$ & & $8.7 \cdot 10^{-3}$ & $5.6 \cdot 10^{-3}$ & & $7.3 \cdot 10^{-3}$ & $4.9 \cdot 10^{-3}$ & \\
\hline
\end{tabular}

\begin{tabular}{|c|c|c|c|c|c|c|c|c|}
\hline \multicolumn{9}{|c|}{ Compound 2 ( $\beta^{\prime}, \beta^{\prime}$ dimer) } \\
\hline \multicolumn{3}{|c|}{ Experimental[a] } & \multicolumn{3}{|c|}{ Calc. TDDFT (transoid conformer) ${ }^{[\mathrm{b}, \mathrm{d}]}$} & \multicolumn{3}{|c|}{ Calc. CC2 (transoid conformer) ${ }^{[b, d]}$} \\
\hline Absorption & Emission & Stokes shift & Absorption & Emission & Stokes shift & Absorption & Emission & Stokes shift \\
\hline $\begin{array}{c}552 \mathrm{~nm} \\
(2.24 \mathrm{eV})\end{array}$ & $\begin{array}{c}603 \mathrm{~nm} \\
(2.05 \mathrm{eV})\end{array}$ & \multirow{3}{*}{$\begin{array}{c}51 \mathrm{~nm} \\
(0.19 \mathrm{eV})\end{array}$} & $\begin{array}{c}469 \mathrm{~nm} \\
(2.64 \mathrm{eV})\end{array}$ & $\begin{array}{c}521 \mathrm{~nm} \\
(2.38 \mathrm{eV})\end{array}$ & \multirow{3}{*}{$\begin{array}{c}52 \mathrm{~nm} \\
(0.26 \mathrm{eV})\end{array}$} & $\begin{array}{c}477 \mathrm{~nm} \\
(2.59 \mathrm{eV})\end{array}$ & $\begin{array}{c}516 \mathrm{~nm} \\
(2.40 \mathrm{eV})\end{array}$ & \multirow{3}{*}{$\begin{array}{c}39 \mathrm{~nm} \\
(0.19 \mathrm{eV})\end{array}$} \\
\hline$g_{\mathrm{abs}}$ & $g_{\text {lum }}$ & & $g_{\mathrm{abs}}$ & $g_{\text {lum }}$ & & $g_{\mathrm{abs}}$ & $g_{\text {lum }}$ & \\
\hline $5.0 \cdot 10^{-4}$ & $4 \cdot 10^{-4}$ & & $3.8 \cdot 10^{-4}$ & $1.2 \cdot 10^{-4[f]}$ & & $8.0 \cdot 10^{-4}$ & $2.1 \cdot 10^{-4}$ & \\
\hline & & & \multicolumn{3}{|c|}{ Calc. TDDFT (cisoid conformer) ${ }^{[b, c]}$} & \multicolumn{3}{|c|}{ Calc. CC2 (cisoid conformer) $)^{[b, d]}$} \\
\hline & & & Absorption & Emission & Stokes shift & Absorption & Emission & Stokes shift \\
\hline & & & $\begin{array}{c}467 \mathrm{~nm} \\
(2.66 \mathrm{eV})\end{array}$ & $\begin{array}{c}528 \mathrm{~nm} \\
(2.35 \mathrm{eV})\end{array}$ & \multirow{3}{*}{$\begin{array}{c}61 \mathrm{~nm} \\
(0.31 \mathrm{eV})\end{array}$} & $\begin{array}{c}478 \mathrm{~nm} \\
(2.59 \mathrm{eV})\end{array}$ & $\begin{array}{c}521 \mathrm{~nm} \\
(2.38 \mathrm{eV})\end{array}$ & \multirow{3}{*}{$\begin{array}{c}43 \mathrm{~nm} \\
(0.21 \mathrm{eV})\end{array}$} \\
\hline & & & $g_{\text {abs }}$ & $g_{\text {lum }}$ & & $g_{\text {abs }}$ & $g_{\text {lum }}$ & \\
\hline & & & $8.3 \cdot 10^{-4}$ & $3.4 \cdot 10^{-4[f]}$ & & $1.8 \cdot 10^{-3}$ & $3.1 \cdot 10^{-4}$ & \\
\hline
\end{tabular}

[a] In dichloromethane solution. Absorption and emission maxima are listed and Stokes shift estimated thereof. [b] M06-2X/def2-TZVP level in vacuo. [c] SCSCC2/def2-SVP level in vacuo. [d] SCS-CC2/def2-TZVP level in vacuo. [e] Calculated on model 2a. [f] Calculated sign is opposite to the experiment (see the text).

In addition to the $g_{\text {lum }}$ value, the corresponding absorption dissymmetry factor ( $\left.g_{\text {abs }}\right)$ should be considered, also known as Kuhn's $g$-factor. It is defined as:

$$
g_{\mathrm{abs}}=\frac{\mathrm{A}_{\mathrm{L}}-\mathrm{A}_{\mathrm{R}}}{\frac{1}{2}\left(\mathrm{~A}_{\mathrm{L}}+\mathrm{A}_{\mathrm{R}}\right)}=\frac{\varepsilon_{\mathrm{L}}-\varepsilon_{\mathrm{R}}}{\frac{1}{2}\left(\varepsilon_{\mathrm{L}}+\varepsilon_{\mathrm{R}}\right)}=\frac{\Delta \varepsilon}{\varepsilon}
$$

where $A_{L}$ and $A_{R}$ are the absorbance for left and right polarized light and similarly $\varepsilon_{L}$ and $\varepsilon_{R}$ are the molar extinction coefficients of the two polarizations. It can be observed from Table 1 that $g_{\text {lum }}$ and $g_{\text {abs }}$ are roughly comparable for 1 (both in the order of $\left.10^{-3}\right)$. This indicates that the phenomena responsible for the two chiroptical properties, namely absorption (ECD) and emission $(\mathrm{CPL})$, are consistent with each other.

The experimental CPL spectra of compound 2 (Table 1 and Figure 2) shows a weaker signal by an order of magnitude than compound 1, with a $g_{\mathrm{lum}}= \pm 4 \cdot 10^{-4}$ (positive for $(\mathrm{a} R)-2$ and negative for (aS)-2). The Stokes shift is also smaller, although still very large, amounting to $51 \mathrm{~nm}(0.19 \mathrm{eV})$. Again, the sign and the magnitude of the $g_{\text {lum }}$ are roughly comparable with the corresponding $g_{\text {abs }}$ values and any effect of light re-absorption and circular dichroism on the CPL spectrum can be neglected. In particular, the CPL sign is consistent again with the sign of the most red-shifted ECD band, thus the apparent violation of the exciton chirality rule mentioned in the Introduction seems to be valid also for the emission. In the case of compound 2 , the fluorescence spectrum is slightly tailed towards long wavelengths, suggesting the presence of two distinct emission bands; as we will discuss below, they are related to the contribution from different conformers.
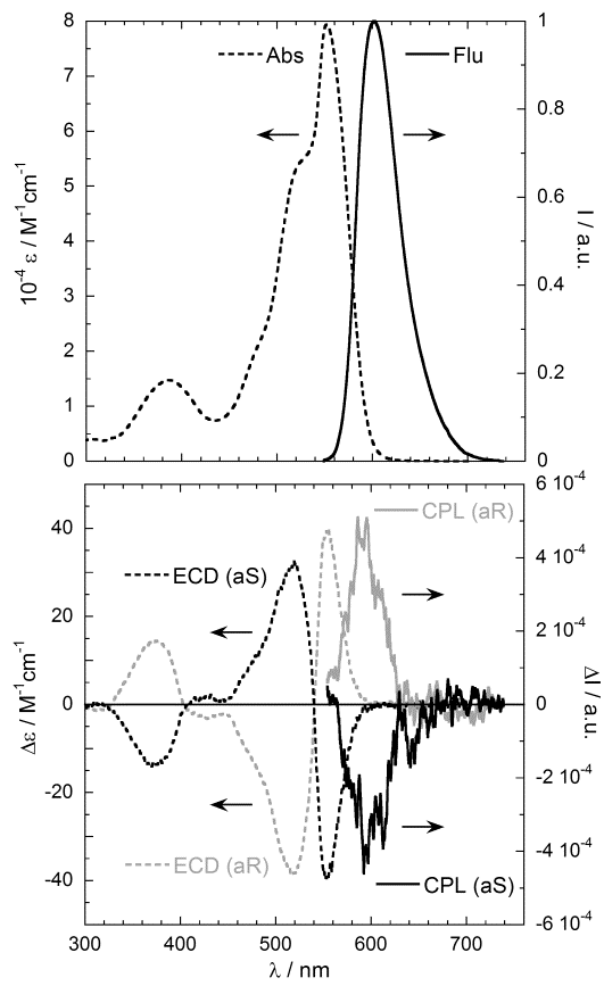

Figure 2. Experimental absorption and fluorescence spectra (top), ECD and CPL spectra (bottom) of compound 2. The scale of the CPL is normalized to the maximum of emission, so that the glum values can be directly read on $y$ axis at the maximum of each curve. Note the different ECD and CPL scales with respect to Figure 1. 


\section{Excited state and CPL spectra simulations}

Extensive studies of the excited states of BODIPY dyes have been recently performed by Le Guennic, Jacquemin and

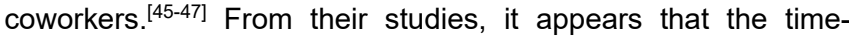
dependent density functional theory (TDDFT) is able to reproduce absorption and emission spectra of these compounds In particular the global hybrid M06-2X functional of Truhlar's "M06 family"[48] performs especially well in combination with a basis set of triple- $\zeta$ quality. For this reason, the M06-2X/def2TZVP combination was used in our study. Other functionals (CAM-B3LYP, $\omega B$ B7X-D) were also tested for comparison; the data obtained at CAM-B3LYP/def2-TZVP level are reported in the Supporting Information (Table S1).

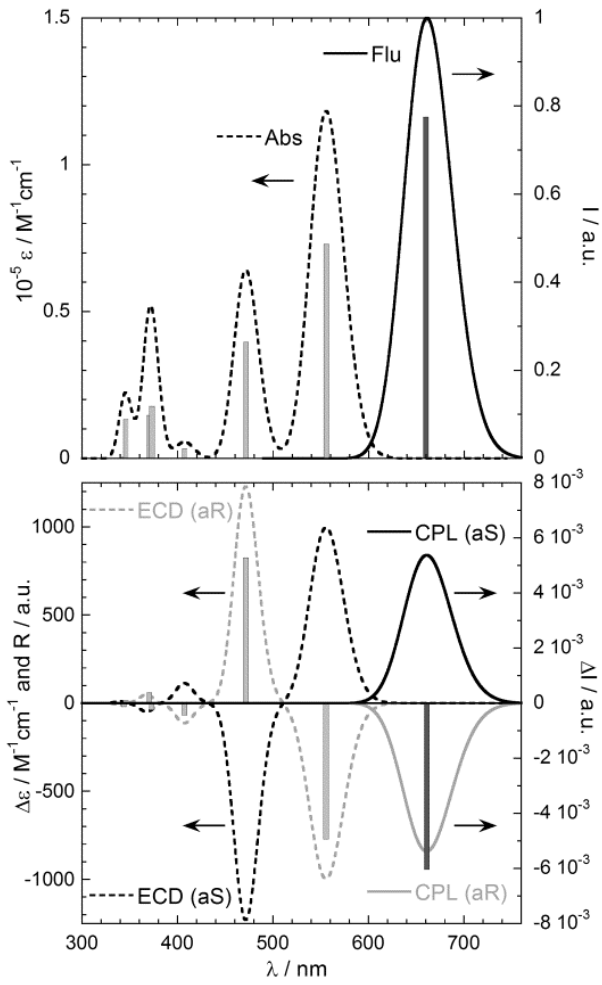

Figure 3. Calculated absorption and fluorescence spectra (top), ECD and CPL spectra (bottom) of compound 1. Calculations run at M06-2X/def2-TZVP level in vacuo, spectra generated as sums of Gaussians with $\sigma=0.1 \mathrm{eV}$, shifted by $-0.4 \mathrm{eV}$. The scale of the CPL is normalized with the same procedure used for experimental spectra. Vertical bars represent oscillator and rotational strengths (in arbitrary absolute units).

Calculated absorption and ECD spectra of compounds 1 and 2 have been reported before at CAM-B3LYP/6-311+G* and RISCS-CC2/def2-SVP levels. ${ }^{[27]}$ In Table 1 and Figures 3 and 4, we show the results of the calculations at the M06-2X/def2TZVP level (compound $\mathbf{2}$ is replaced here by the truncated model 2a, Scheme 1), which confirm the already observed trends. M06-2X calculations reproduce very well both absorption and ECD spectra, apart from a systematic energy shift which amounts to $0.4 \mathrm{eV}$ at the current level of calculation. For compound 1 , a single energy minimum is found with $\mathrm{N}-\mathrm{C} 3-\mathrm{C} 3$ '-N dihedral of $105^{\circ}$ (Figure 5, dark grey). The potential energy associated with the aryl-aryl torsional mode is a sharp curve with high barriers (Supporting Information, Figure S2), due to steric and electronic repulsion between the $\mathrm{BF}_{2}$ groups and the 2/2'-
$\mathrm{CH}_{3}$ groups. For the other isomer, 2a, such contacts are removed so that a larger torsional motion is allowed around the aryl-aryl axis. As a consequence, two shallow energy minima are obtained (Figure 6, dark grey) with C8a-C1-C1'-C8a' dihedral angles of $52^{\circ}$ (cisoid conformer) and $128^{\circ}$ (transoid conformer) separated by a barrier of less than $3 \mathrm{kcal} / \mathrm{mol}$ along a flat potential energy well (Figure 7 , bottom curve).

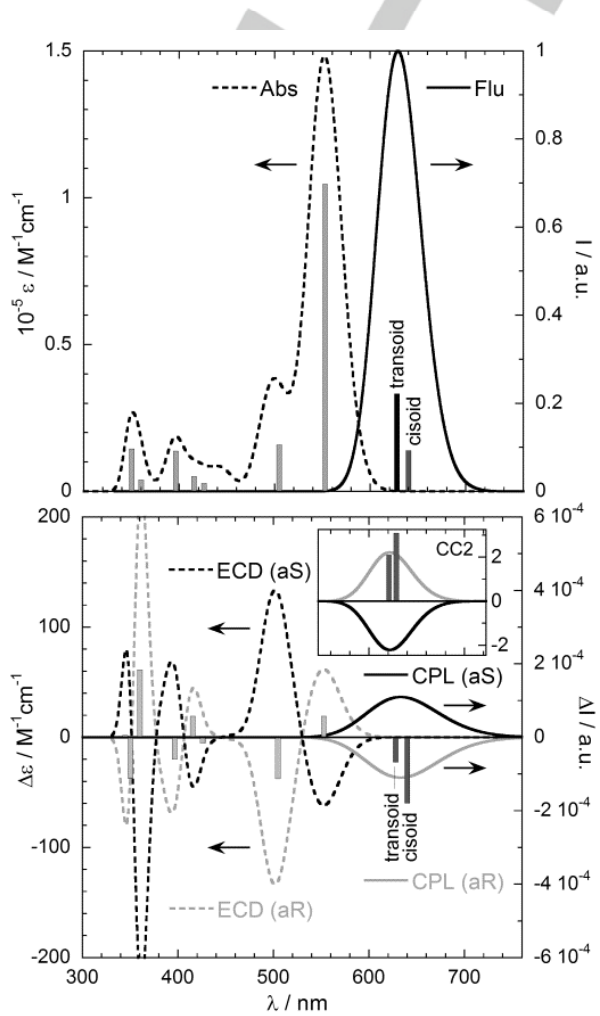

Figure 4. Calculated absorption and fluorescence spectra (top), ECD and CPL spectra (bottom) of model compound 2a. Calculations run at M06-2X/def2TZVP level in vacuo, spectra generated as sums of Gaussians with $\sigma=0.1 \mathrm{eV}$, shifted by $-0.4 \mathrm{eV}$. The scale of the CPL is normalized with the same procedure used for experimental spectra. Vertical bars represent oscillator and rotational strengths (in arbitrary absolute units), shown only for the transoid conformer in the case of absorption and ECD spectra. Calculated spectra are the Boltzmann-weighted averages for the two conformers (cisoid and transoid), using populations estimated from relative free energies at $300 \mathrm{~K}$. The inset shows CPL spectra calculated at CSC-CC2/def2-TZVP level in vacuo; the horizontal axis is aligned to the main axis, while the vertical axis is offset and the labels are $10^{-4} \mathrm{\Delta l} / \mathrm{a} . \mathrm{u}$.

All the computational procedures tested consistently predict the cisoid conformer to be the more stable one according to internal energies, but the less stable according to free energies. On the basis of these latter values, the transoid conformer has a population of $80 \%$ to $98 \%$ at $300 \mathrm{~K}$, depending on the method. An independent source of information, namely VCD spectra, confirms that the transoid isomer is largely dominant at room temperature. ${ }^{[27]}$ Thus in this specific case, the calculated free energies appear to be more accurate than internal energies. Although this is expected in principle, it is not always true in practice, because of the inaccurate estimation of vibrational entropy terms associated with low-frequency modes.\{Grimme, 2008 \#91\} \{Pescitelli, 2016 \#76\} Therefore, the calculated spectra for model $\mathbf{2 a}$ shown in Figure 4 were obtained after a 
Boltzmann averaging based on free energies. However, for such a low interconversion barrier the standard Boltzmann averaging procedure is questionable and a vibrational averaging should, more accurately, be considered. ${ }^{[49-50]}$

The DFT-optimized conformations of compounds 1 and $2 a$ can be checked against available X-ray structures, although it must be stressed that for flexible biaryls the $\mathrm{X}$-ray geometries may largely differ from solution or gas-state ones.\{Di Bari, 1999 \#93;Barich, 2001 \#94;Shi, 2015 \#95\} For compound 1 no X-ray structure is available, however two 6,6'-di-p-tolyl analogs of 1 were considered for comparison.\{Bröring, 2008 \#33;Bröring, $2010 \# 88\}$ They show N-C3-C3'-N' dihedrals in the range 92$97^{\circ}$ and an overall arrangement similar to that calculated for 1 . The X-ray structure of compound $\mathbf{2}$ had been determined previously\{Ahrens, $2013 \# 92\}$ and is reported in the Supporting Information (Figure S4).\{NOTE:CCDC 1492720 contains the supplementary crystallographic data for compound $\mathbf{2}$. These data are provided free of charge by The Cambridge Crystallographic Data Centre via http://www.ccdc.cam.ac.uk/\} It features C8a-C1-C1'-C8a' dihedral angles of $46^{\circ}$ and $51^{\circ}$ (two independent molecules) which compare well with our calculated structure for the cisoid conformer.

For both compounds $\mathbf{1}$ and $\mathbf{2}$ (or its model 2a), absorption and ECD spectra (Figures 1 and 2) compare very well with simulated ones (Figures 3 and 4). In particular, the sign of the ECD couplet around $500 \mathrm{~nm}$ is reproduced by the calculations also for compound 2, for which the exciton chirality rule fails as mentioned above and discussed previously. ${ }^{[27]}$ A positive ECD couplet is experimentally found and consistently predicted by TDDFT for the (aS) enantiomer of compound 1 and the $(a R)$ enantiomer of compound 2. More interestingly, the calculated dissymmetry factors $g_{\mathrm{abs}}$ are also in excellent agreement with the experimental values for both compounds (see Table 1, where the transoid conformer should be considered for model $\mathbf{2 a}$ ).

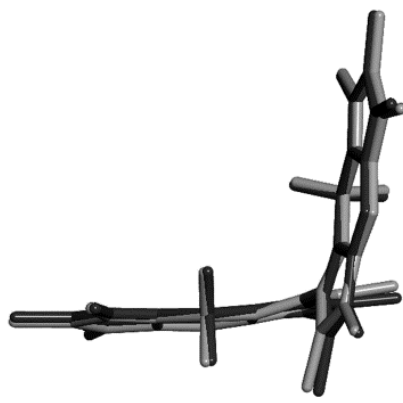

Figure 5. Overlap of ground state (dark grey) and excited state (light grey) geometries calculated at M06-2X/def2-TZVP level for compound (aR)-1. Hydrogen atoms removed for clarity.

(a)
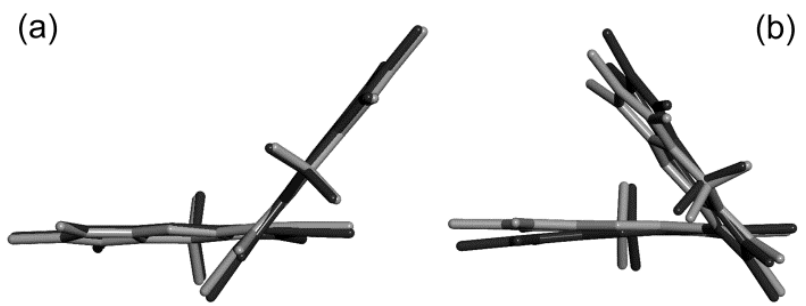

Figure 6. Overlap of ground state (dark grey) and excited state (light grey) geometries calculated at M06-2X/def2-TZVP level for model compound (aR)- 2a in the transoid (a) and cisoid (b) conformation. Hydrogen atoms removed for clarity.

The calculations of emission and CPL spectra were run relying on a well-established computational procedure developed by Pecul and Ruud and by Abbate, Longhi and coworkers. ${ }^{[36,51-55]}$ The procedure is based on the optimization of the molecule in its first singlet excited state geometry, and on the evaluation of the excited states obtained thereof. Such a procedure has a general validity as it has been applied to various organic compounds, ranging from simple ketones and enones\{Pecul, 2011 \#68\} \{Longhi, $2013 \# 7\}$ to e.g. helicenes\{Longhi, 2016 \#10;Abbate, $2014 \# 12\}$ and oligothiophenes.\{Longhi, 2014 \#63\} Our calculations were run in vacuo at the already mentioned M062X/def2-TZVP level. As far as compound 1 is concerned, this procedure led to very satisfying results (Figure 3 and Table 1): the calculated Stokes shift is $0.35 \mathrm{eV}$, in excellent agreement with the experimental value of $0.32 \mathrm{eV}$; the predicted sign of the CPL band is in agreement with both the first calculated ECD band and the experiment, that is, positive for enantiomer (aS)-1; the computed value for $g_{\text {lum }}$ is also very close to the experimental value (5.6 vs $3.8 \cdot 10-3)$. The good agreement confirms the validity of the current computational approach in a case devoid of any conformational ambiguity. The excited-state geometry of compound $(a R)-1$ is overlapped in Figure 5 (light grey) with the ground-state one. Both BODIPY rings appear more distorted in the excited state as a consequence of two opposite forces, namely the propensity to augment conjugation (see the results for $2 \mathrm{a}$ below) and the resulting steric contact between the $\mathrm{BF}_{2}$ group on one ring and the $2-\mathrm{CH}_{3}$ group on the other ring. The relatively large difference between the ground and excited structures (the RMS deviation between the heavy atoms for each ring is $0.10 \AA$ ) is one reason for the large Stokes shift.

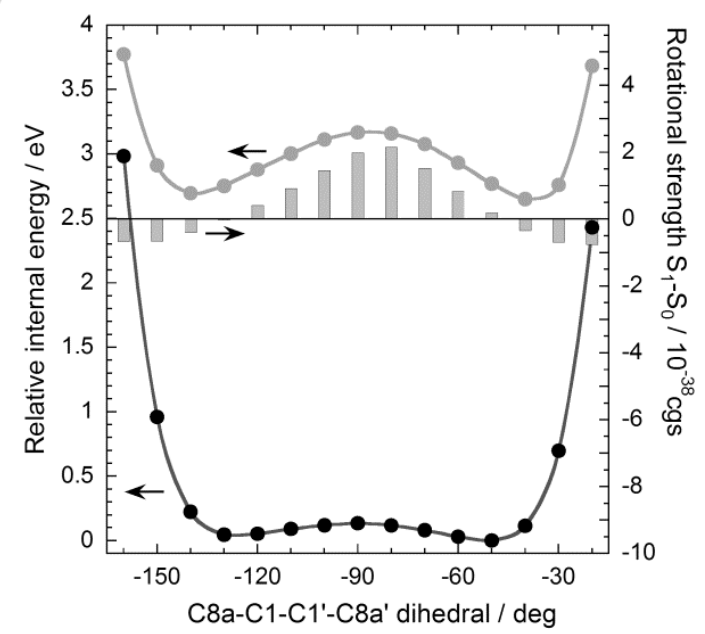

Figure 7.. Torsional energy scans around the aryl-aryl axis for the ground state (lower black curve) and the first excited state (upper grey curve) for model compound $(\mathrm{a} R)-\mathbf{2} \mathbf{a}$, and corresponding calculated rotational strengths for the excited-to-ground state emission (vertical bars). Each curve is the results of two non-relaxed scans run at M06-2X/def2-TZVP level using the transoid structures (depicted in Figure 6a) as starting geometries for dihedrals $<-90^{\circ}$ and the cisoid structures (depicted in Figure $6 \mathrm{~b}$ ) as starting geometries for dihedrals $\geq-90^{\circ}$. The internal energies relative to the lowest-energy structure (ground state cisoid conformer) are plotted. 
The situation for compound $\mathbf{2}$ is much more complicated because of the rotational freedom. Two excited-state energy minima are obtained for model $\mathbf{2} \mathbf{a}$, again with cisoid and transoid conformation. In accord with a known tendency of biaryl compounds, ${ }^{[56]}$ this BODIPY dimer relaxes toward a more planar geometry in the first excited state. For the cisoid conformer, in fact, the C8a-C1-C1'-C8a' dihedral decreases from $52^{\circ}$ to $40^{\circ}$, while for the transoid one, it increases from $128^{\circ}$ to $136^{\circ}$ (Figure 6 , light grey). In both cases, the planarity of each ring is almost unaffected (the RMS deviation between the heavy atoms for each ring is $0.05 \AA$ for both conformers). In the excited state, the torsional energy curve shows two minima separated by a barrier as high as $10 \mathrm{kcal} / \mathrm{mol}$ (upper curve in Figure 7). Full optimization of the maximum-energy excited-state conformer (with C8a-C1-C1'-C8a' dihedral $\approx 90^{\circ}$ ) lowers the barrier to about $7 \mathrm{kcal} / \mathrm{mol}$, which is still high enough to prevent any transoid-to-cisoid conversion during the excited state lifetime. In this situation, we may assume that the two excited-state minima are populated in a measure proportional to the population of the respective ground state conformers. In principle, the calculated stationary geometries for the excited state might actually be saddle-points along a double-well surface, originated from the coupling of two degenerate states in the quasi- $\mathrm{C}_{2}$ symmetry, rather than true minima. ${ }^{[57]}$ To exclude this possibility, we reoptimized the geometry of both conformers after manual distortion of the BODIPY rings (i.e., one ring was compressed and one elongated along the major axis direction, and the arylaryl dihedral varied). These optimizations converged to the same minima found before.

Starting from the discussed excited-state geometries of model compound $2 \mathbf{a}$, TDDFT calculations were run using the same approach followed for compound 1. The calculated Stokes shift for $2 a$ is $0.26 \mathrm{eV}$ for the transoid conformer and $0.31 \mathrm{eV}$ for the cisoid conformer. Taking into account the conformer populations discussed above, the overall simulated emission profile is a curve tailed toward the long wavelengths, similarly to the experiment (Figure 4). The calculated Stokes shifts and $g_{\text {lum }}$ values for the dominating transoid conformer turn out to be both very close to the experimental values (see Table 1). However, the sign of the calculated CPL band is opposite to both the first calculated ECD band and the experimental CPL band (Figure 4). Thus, enantiomer $(a R)-2$ shows a positive ECD couplet and positive CPL band, while the predicted CPL band for model $(a R)-2 a$ is negative. This inconsistency occurs for both conformers. Thus, the TDDFT-based computational approach fails to reproduce the CPL spectrum of compound $\mathbf{2}$ when the recommended M06-2X functional is employed.

In the attempt to improve the results of TDDFT calculations we first tried other range-separated functionals, namely CAMB3LYP and $\omega-B 97 X D$, which are known to be very accurate in the calculations of chiroptical properties. ${ }^{[58]}$ Both CAMB3LYP/def2-TZVP and $\omega$-B97XD/def2-TZVP calculations however predicted again the wrong sign for the critical CPL band (the whole set of data from CAM-B3LYP/def2-TZVP calculations are reported in the Supporting Information, Table S1; some data from $\omega-B 97 X D /$ def2-TZVP are also shown in the Supporting Information). Next, M06-2X/def2-TZVP calculations were performed including a solvent model for dichloromethane by using the state-specific vertical excitation model (VEM). ${ }^{[59]}$ The results obtained (not shown) were very similar to those in vacuo, apart from a small bathochromic shift. Similarly, representative calculations run on the whole compound 2 , rather than on its model $2 \mathbf{a}$, did not solve the problem.

Therefore, the entire set of calculations was run at SCS-CC2 level of theory, employing DFT- and TDDFT-optimized geometries for ground and excited-state SCS-CC2 calculations, respectively, and using the def2-SVP and def2-TZVP basis sets for compounds 1 and $\mathbf{2 a}$, respectively. Pecul and Ruud used a similar calculation approach for the CPL spectrum of a $\beta, \gamma-$ enone, ${ }^{[51]}$ while more recently Crawford et al. applied EOMCCSD (equation-of-motion CC singles and doubles) CPL calculations to a series of ketones and enones. ${ }^{[60]}$ The results of SCS-CC2 calculations on compounds 1 and $2 \mathrm{a}$ are summarized in Table 1. Apart from the CPL sign, the agreement between SCS-CC2 theory and experimental data is as good as TDDFT for compound 1, and slightly better than TDDFT for compound 2 (and its model $\mathbf{2 a}$ ) in terms of relative transition energies (Stokes shift) and $g$-values. Most importantly, the calculated CPL spectrum for compound $\mathbf{2 a}$ has now the correct sign in keeping with the experiment, namely the predicted CPL band for model $(\mathrm{a} R)-2 \mathrm{a}$ is positive (see inset in Figure 4, bottom). It is noteworthy that in all cases described by Pecul and Ruud and Crawford et al., RI-CC2 or EOM-CCSD calculations always agreed with TDDFT on the predicted sign of CPL bands. ${ }^{[51,60]}$ The present inconsistency between TDDFT and coupled-cluster calculations is, to the best of our knowledge, unprecedented in the context of CPL, while in the context of ECD calculations some discrepancies between TDDFT and CC2 have been reported, mostly related to transitions with large charge-transfer character. ${ }^{[61-62]}$

The observation that $\mathrm{CC} 2$ calculations could reproduce the experimental CPL of compound 2, while TDDFT calculations with range-separated or hybrid meta-GGA functionals with around $50 \%$ of exact exchange could not, suggested that a crucial role may be played by the fraction of exact (or HartreeFock) exchange included in the TDDFT functionals. To test this hypothesis, we turned our attention to Truhlar's M06-HF functional that includes $100 \%$ of exact exchange. ${ }^{[63]}$ This functional does not perform as well as e.g. M06-2X for predicting valence excitations, however it is known to outperform several other functionals for predicting long-range charge transfer excitations. ${ }^{[48]}$ Therefore we ran TDDFT calculations on compounds 1 and 2a at M06-HF/def2-TZVP level, employing for the ground and excited states the respective geometries optimized at M06-2X/def2-TZVP level. The calculation results are shown in the Supporting Information, Table S1. Similarly to CC2, M06-HF calculations also recovered the correct sign for the critical CPL band of compound 2. In fact the predicted sign was positive for model $(\mathrm{a} R)-\mathbf{2} \mathbf{a}$ in the dominant transoid conformation, and negative but almost negligible for the minor cisoid conformer. Overall, the agreement between calculated and experimental data in terms of relative transition energies (Stokes shift) and $g$-values is better than with M06-2X and slightly poorer than with $\mathrm{CC} 2$.

In conclusion, only the two methods based on an exact evaluation of the exchange term - CC2 and M06-HF - did predict the correct sign for the CPL band of compound 2, while all other DFT-based methods with various fractions of $\mathrm{HF}$ exchange predicted the wrong sign. A correct evaluation of the electron density delocalization seems to be a necessary requisite to reproduce the chiroptical emission behavior of the BODIPY DYEmer 2. A detailed investigation of the influence of 
the exact exchange to simulate the emission properties of this type of compounds is currently underway and will be the subject of a future work.

Apart from the role played by the exact exchange, are there any specific reasons for the failure of three different hybrid functionals (M06-2X, CAM-B3LYP and $\omega$-B97XD) for compound 2? This failure seems to be strictly related to the intrinsic non-robustness of the CPL band measured on 2 and calculated on $2 \mathbf{a}$. Where the prediction of chiroptical properties is concerned, an estimation of the robustness of calculations (and of the corresponding measurements) is possible by evaluating the angle $\xi$ between the calculated electric and magnetic dipole moments $\mu$ and $\mathbf{m}^{\text {[64-65] }}$ All chiroptical properties are in fact allied with a quantity called rotational strength, defined as the scalar product between $\mu$ and $\mathbf{m}^{\text {[66] }}$ The larger is the deviation of $\xi$ from $90^{\circ}$, the more robust is the prediction of the rotational strength. In the case of $\mathrm{S}_{1}-\mathrm{S}_{0}$ emission calculations for $(R)-\mathbf{2} \mathbf{a}$, the angle $\xi$ between the electric and magnetic dipole moments is very close to $90^{\circ}$, i.e. the value where the rotational strength vanishes. The robustness angle $\xi$ is indeed between $87^{\circ}$ and $93^{\circ}$ for both transoid and cisoid conformers with every employed functional (values are listed in Table S2, Supporting Information). In comparison, the corresponding ECD band for the $\mathrm{S}_{0}-\mathrm{S}_{1}$ excitation of $(R)-2 \mathrm{a}$ is much more robust, with $\xi$ angles between $75^{\circ}$ and $81^{\circ}$ (with def2-TZVP basis set). The situation is much less critical in isomer 1, for which both ECD and CPL bands appear very robust because $\xi$ angles are $\approx 132^{\circ}$ and $123^{\circ}$ $125^{\circ}$, respectively.
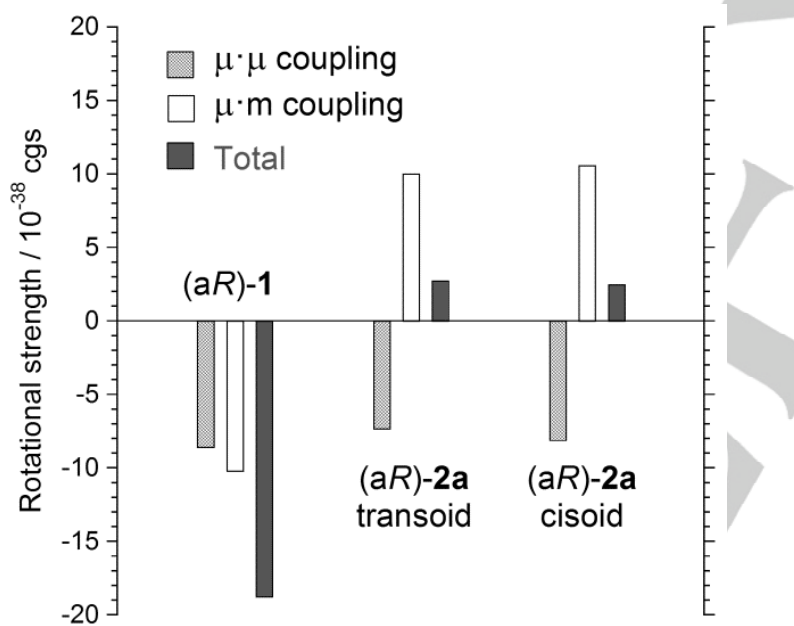

Figure 8. Rotational strengths calculated for the $S_{0}-S_{1}$ excitation of compounds (S)-1 and (aR)-2a (two conformers) at M06-2X/TZVP level using geometries optimized with M06-2X/def2-TZVP. The calculations were run with the tool EXAT which disentangles the contributions from $\mu-\mu$ and $\mu-m$ couplings. The values compare well with previous results obtained with a preliminary version of the software and at a different level of theory. ${ }^{[27]}$

The differences between the calculated robustness angles $\xi$ for 1 and $2 a$ correlate well with the differences seen in the experimental spectra. They can also be interpreted using an excitonic picture, because both ECD and CPL spectra of $\mathbf{1}$ and $\mathbf{2}$ are dominated by the already discussed exciton coupling mechanism. This exciton coupling is due to the combination of two distinct contributions, namely the electric-electric $(\mu-\mu)$ and the electric/magnetic ( $\mu-\mathrm{m})$ couplings. ${ }^{[27,29]}$ The main difference between 1 and 2 lies in the way the two contributions combine with each other. Figure 8 shows the values estimated for the $\mu-\mu$ and $\mu-m$ couplings for compounds (S)-1 and (R)-2a (two conformers), using the tool EXAT ${ }^{[67]}$ and TDDFT calculations at M06-2X/TZVP level for each monomer. The details of this kind of approach have been reported previously. ${ }^{[29]}$ For the $S_{0}-S_{1}$ excitation of isomer $1, \mu-\mu$ and $\mu-m$ couplings have the same sign and reinforce each other, thus the resulting ECD bands are relatively strong. On the contrary, for model compound $2 a$, the $\mu$ $\mu$ and $\mu-m$ couplings have opposite sign, mutually cancel each other, and the resulting ECD bands are weak. This is true for both the transoid and the cisoid conformers. A similar phenomenon seems to occur in emission, where the CPL band of $\mathbf{1}$ is one order of magnitude more intense than 2 . This is found both experimentally and theoretically (see Table 1 and Figures 1-4), and is reflected in the above-mentioned values of angle $\xi$ which are very close to $90^{\circ}$ for model 2 a.

A second reason for the weak CPL band of the BODIPY DYEmer 2, which concurs with the previous discussed one, is related to the conformational flexibility. In fact, the sign and intensity of the predicted CPL band for model $2 \mathbf{a}$ at M062X/def2-TZVP level depend on the aryl-aryl torsion as shown in Figure 7 (vertical bars). For the enantiomer $(a R)-2 \mathbf{a}$, the band is negative and small for the transoid excited-state minimum with C8a-C1-C1'-C8a' dihedral of $-136^{\circ}$, however it vanishes around $-130^{\circ}$ and is positive at $-120^{\circ}$. A similar situation occurs for the cisoid conformer: the band is negative and small for a C8a-C1C1'-C8a' dihedral of $-40^{\circ}$, but it turns to positive already at $-50^{\circ}$. Thus, this CPL band is also non-robust with respect to small geometrical distortions. In particular, it vanishes and changes sign within each of the two energy wells associated with the excited-state minima for the aryl-aryl torsional mode. In the ground state, conversely, the predicted first ECD band is always positive for $(a R)-2 a$ along a large portion of the torsional energy curve, in particular that comprised between and including the two minima (Supporting Information, Figure S3). A pronounced dependence of CPL on the aryl-aryl torsion has been reported for other biaryls, for example 1,1'-binaphthyl derivatives. ${ }^{[33,68-69]}$ In these cases, however, the observed CPL bands are allied with weak transitions of the naphthalene ${ }^{1} \mathrm{~L}_{b}$-type, and do not belong to the main exciton couplet between naphthalene ${ }^{1} B_{b}-$ type transitions, in contrast with BODIPY DYEmers. In the present situation, it is also likely that the dependence of the magnetic and electric transition dipoles on the torsional motion cannot be disregarded. The introduction of such effect with a fully quantum approach cannot be accomplished at a pure electronic level and it would require the inclusion of vibronic contributions beyond the Franck-Condon approximation. ${ }^{[70-72]}$.

\section{Conclusions}

With our current experimental set-up, it is possible to record CPL spectra with excellent signal-to-noise ratio and to reliably measure signals at least down to $g_{\mathrm{lum}} \approx 10^{-4}$.

Our results show that for compound $\mathbf{1}$, which exists as a single minimum in both the ground-state and excited-state, it is possible to accurately reproduce the features of both the ECD and CPL spectra (including the absorption and emission dissymmetry factors), using a consolidated calculation method based on the M06-2X functional recommended for BODIPY's. On the other hand, compound $\mathbf{2}$ is associated with weaker ECD 
and CPL spectra, and it is endowed with large flexibility in both the ground and excited states. In this case several hybrid and range-separated functionals, such as M06-2X, CAM-B3LYP and $\omega B 97 X-D$, failed to reproduce the sign of the CPL spectra. Only methods based on an exact evaluation of the exchange term such as M06-HF and SCS-CC2 correctly predicted the CPL spectra. In our interpretation, the failure of the former methods is due to two concomitant factors. First, a peculiar combination of electric/electric and electric/magnetic exciton couplings makes the rotational strength intrinsically non-robust for $\mathbf{2}$. Second, the calculated CPL band is very sensitive to the excited-state conformation and it varies from one sign to another for geometries close to the predicted excited-state minimum.

Our results may contribute to rationalize the features required for the development of single organic molecules with highly polarized emission, as required for applications in chiral optoelectronics and photonics.

\section{Experimental Section}

\section{Materials}

The synthesis, enantioseparation and full characterization of compounds 1 and 2 has been reported previously. ${ }^{[27,40]}$

\section{$X$-ray analysis}

Suitable crystals of 2 were obtained by crystallization from methanol. $X$ Ray intensity data was collected at 100(2) K using an Oxford Diffraction Nova A $(\mathrm{CuK} \alpha)$ instrument. A single crystal was mounted in inert perfluoroether oil on top of a glass fiber. The structure was solved by direct methods with SHELXS-97.\{Sheldrick, 2015 \#96\} Refinements were carried out by full-matrix least squares techniques against all F2 using SHELXL-2014/7. \{Sheldrick, 2015 \#96\}

Crystal data for 2: $\mathrm{C}_{30} \mathrm{H}_{36} \mathrm{~B}_{2} \mathrm{~F}_{4} \mathrm{~N}_{4} \times 0.75 \mathrm{CH}_{4} \mathrm{O}, 574.28$, triclinic, space group $\mathrm{P} \overline{1}, a=13.899(2) \AA, b=14.3413(15) \AA, c=18.6238(16) \AA, \alpha=$ 96.435(7) $)^{\circ}, \beta=104.204(9)^{\circ}, \gamma=117.404(11)^{\circ}, V=3083.9(7) \AA^{3}, Z=4$, $\rho_{\text {calc }}=1.237 \mathrm{~g} \mathrm{~cm}^{-3}, \mu(\mathrm{Cu}-\mathrm{K} \alpha)=0.750 \mathrm{~mm}^{-1}, \mathrm{R}_{1}[\mathrm{I}>2 \sigma(\mathrm{I})]=0.0472$, $w R_{2}$ (all data) $=0.1361$.

\section{Spectroscopy}

Absorption and ECD spectra were measured with a JASCO V-650 Spectrophotometer and a JASCO J-710 spectropolarimeter, respectively, on $5 \cdot 10^{-5} \mathrm{M} \mathrm{CH}_{2} \mathrm{Cl}_{2}$ solutions using a $1 \mathrm{~cm}$ quartz cell. $\mathrm{CPL}$ and fluorescence spectra were recorded on $5 \cdot 10^{-5} \mathrm{M} \mathrm{CH}_{2} \mathrm{Cl}_{2}$ solutions with a home-built instrument. A detailed description of the instrument, inspired from a previous design, ${ }^{[73]}$ is reported in the Supporting Information (Figure S1). The samples were excited with a $90^{\circ}$ geometry using a green LED source $\left(\lambda_{\max }=517 \mathrm{~nm}, \mathrm{HWHM}=15 \mathrm{~nm}\right)$.

\section{Calculations}

All DFT-based computations were run with the Gaussian'09 program. ${ }^{[74]}$ DFT and TDDFT calculations were run with the hybrid M06-2X functional[ ${ }^{[48]}$ and the triple- $\zeta$ basis set with polarization functions def2TZVP. ${ }^{[75]}$ Other functionals (CAM-B3LYP, $\omega B$ 97X-D, M06-HF) and basis sets (TZVP, def2-SVP) were also tested. All calculations were performed in vacuo. Some representative calculations were run including a solvent model for dichloromethane, using a state-specific vertical excitation model (VEM). ${ }^{[59]}$ Ground-state geometries were optimized with DFT at M06-2X/def2-TZVP level and verified as true minima by frequency calculations at the same level. Excited-state calculations were run with TDDFT at M06-2X/def2-TZVP level. Fluorescence and CPL spectra were normalized at the maximum of fluorescence. In this way, the CPL spectra reflect immediately the corresponding glum values. ${ }^{[53]}$ SCS-CC2 (spincomponent scaling approach for second-order approximate coupledcluster) calculations ${ }^{[76-77]}$ were done with Turbomole ${ }^{[78-79]}$ applying the resolution-of-identity (RI) approximation. For compound 1 the def2-SVP basis set was used while for $2 \mathbf{a}$ the def2-TZVP basis set was used. All spectra were obtained as sums of Gaussians with $\sigma=0.1 \mathrm{eV}$ exponential half-width. Calculated spectra were shifted by $0.4 \mathrm{eV}$ to the red in the comparison with experimental spectra. Excitonic ECD and CPL simulations were performed by using the tool EXAT $^{[67]}$ in the velocity gauge, as detailed in ref. ${ }^{[29]}$.

\section{Acknowledgements}

Ettore Castiglioni gave us constant advice on CPL instrument but he passed away before being able to see it operating. We shall miss his encouraging and enlightening suggestions and criticism. Dr Fabrizio Santoro is acknowledged for useful discussions. Ministero per l'Istruzione, l'Università e la Ricerca (MIUR, PRIN2012, prot. 2012A4Z2RY) and the Deutsche Forschungsgemeinschaft DFG (BR2010/9-1) are thanked for funding.

Keywords: Boron Dipyrrin; TDDFT calculations; Atropisomeric biaryls; Chiroptical spectroscopy; Luminescence dissymmetry factor.

\section{References}

[1] J. P. Riehl, F. S. Richardson, Chem. Rev. 1986, 86, 1-16.

[2] J. P. Riehl, G. Muller, in Comprehensive Chiroptical Spectroscopy, Vol. 1 (Eds.: N. Berova, P. L. Polavarapu, K. Nakanishi, R. W. Woody), John Wiley \& Sons, Inc., Hoboken, NJ, 2012, pp. 65-90.

[3] E. Peeters, M. P. T. Christiaans, R. A. J. Janssen, H. F. M. Schoo, H. P. J. M. Dekkers, E. W. Meijer, J. Am. Chem. Soc. 1997, 119, 9909-9910.

[4] Y. Yang, R. C. da Costa, D.-M. Smilgies, A. J. Campbell, M. J. Fuchter, Adv. Mater. 2013, 25, 2624-2628.

[5] F. Zinna, U. Giovanella, L. D. Bari, Adv. Mater. 2015, 27, 1791-1795

[6] Y. Yang, R. C. da Costa, M. J. Fuchter, A. J. Campbell, Nat. Photon. 2013, 7, 634-638.

[7] C. Wagenknecht, C.-M. Li, A. Reingruber, X.-H. Bao, A. Goebel, Y.-A Chen, Q. Zhang, K. Chen, J.-W. Pan, Nat. Photon. 2010, 4, 549-552.

[8] R. Farshchi, M. Ramsteiner, J. Herfort, A. Tahraoui, H. T. Grahn, Appl. Phys. Lett. 2011, 98, 162508.

[9] C. Wang, H. Fei, Y. Qiu, Y. Yang, Z. Wei, Y. Tian, Y. Chen, Y. Zhao Appl. Phys. Lett. 1999, 74, 19-21.

[10] B. L. Feringa, Acc. Chem. Res. 2001, 34, 504-513.

[11] E. M. Sánchez-Carnerero, A. R. Agarrabeitia, F. Moreno, B. L. Maroto, G. Muller, M. J. Ortiz, S. de la Moya, Chem. Eur. J. 2015, 21, 1348813500

[12] J. Kumar, T. Nakashima, T. Kawai, J. Phys. Chem. Lett. 2015, 6, 34453452

[13] F. Zinna, L. Di Bari, Chirality 2015, 27, 1-13.

[14] F. Zinna, C. Resta, S. Abbate, E. Castiglioni, G. Longhi, P. Mineo, L. Di Bari, Chem. Commun. 2015, 51, 11903-11906.

[15] J. L. Lunkley, D. Shirotani, K. Yamanari, S. Kaizaki, G. Muller, Inorg. Chem. 2011, 50, 12724-12732.

[16] E. Scheler, P. Strohriegl, in Liquid Crystalline Semiconductors: Materials, properties and applications (Eds.: J. R. Bushby, M. S. Kelly, M. O'Neill), Springer Netherlands, Dordrecht, 2013, pp. 197-218.

[17] A. Loudet, K. Burgess, Chem. Rev. 2007, 107, 4891-4932.

[18] G. Ulrich, R. Ziessel, A. Harriman, Angew. Chem. Int. Ed. 2008, 47, 1184-1201.

[19] A. Gossauer, F. Fehr, F. Nydegger, H. Stöckli-Evans, J. Am. Chem. Soc. 1997, 119, 1599-1608. 
[20] E. M. Sánchez-Carnerero, F. Moreno, B. L. Maroto, A. R. Agarrabeitia, M. J. Ortiz, B. G. Vo, G. Muller, S. d. I. Moya, J. Am. Chem. Soc. 2014, 136, 3346-3349.

[21] S. Zhang, Y. Wang, F. Meng, C. Dai, Y. Cheng, C. Zhu, Chem. Commun. 2015, 51, 9014-9017

[22] R. B. Alnoman, S. Rinn, D. C. O'Connor, F. A. Black, B. Costello, P. G. Waddell, W. Clegg, R. D. Peacock, W. Herrebout, J. G. Knight, et al., Chem. Eur. J. 2016, 22, 93-96.

[23] J. F. Kögel, S. Kusaka, R. Sakamoto, T. Iwashima, M. Tsuchiya, R. Toyoda, R. Matsuoka, T. Tsukamoto, J. Yuasa, Y. Kitagawa, et al., Angew. Chem. Int. Ed. 2016, 55, 1377-1381.

[24] H. Lu, J. Mack, T. Nyokong, N. Kobayashi, Z. Shen, Coord. Chem. Rev 2016, in press; DOI 10.1016/j.ccr.2016.1003.1015

[25] C. Ray, E. M. Sánchez-Carnerero, F. Moreno, B. L. Maroto, A. R. Agarrabeitia, M. J. Ortiz, Í. López-Arbeloa, J. Bañuelos, K. D. Cohovi, J. L. Lunkley, et al., Chem. Eur. J. 2016, in press. DOI 10.1002/chem.201601463.

[26] Y. Gobo, M. Yamamura, T. Nakamura, T. Nabeshima, Org. Lett. 2016, in press, DOI 10.1021/acs.orglett.1026b01237.

[27] T. Bruhn, G. Pescitelli, S. Jurinovich, A. Schaumlöffel, F. Witterauf, J. Ahrens, M. Bröring, G. Bringmann, Angew. Chem. Int. Ed. 2014, 53, 14592-14595.

[28] N. Harada, K. Nakanishi, Circular Dichroic Spectroscopy - Exciton Coupling in Organic Stereochemistry, University Science Books, Mill Valley, CA, 1983.

[29] S. Jurinovich, C. A. Guido, T. Bruhn, G. Pescitelli, B. Mennucci, Chem. Commun. 2015, 51, 10498-10501.

[30] K. Tanaka, G. Pescitelli, K. Nakanishi, N. Berova, Monatsh. Chem. 2005, 136, 367-395.

[31] H. Goto, Macromolecules 2007, 40, 1377-1385.

[32] T. Kawai, K. Kawamura, H. Tsumatori, M. Ishikawa, M. Naito, M. Fujiki, T. Nakashima, ChemPhysChem 2007, 8, 1465-1468.

[33] T. Kimoto, N. Tajima, M. Fujiki, Y. Imai, Chem. As. J. 2012, 7, 2836 2841.

[34] J. Kumar, T. Nakashima, H. Tsumatori, T. Kawai, J. Phys. Chem. Lett 2014, 5, 316-321.

[35] T. Nishikawa, N. Tajima, M. Kitamatsu, M. Fujiki, Y. Imai, Org. Biol. Chem. 2015, 13, 11426-11431

[36] G. Mazzeo, S. Abbate, G. Longhi, E. Castiglioni, S. E. Boiadjiev, D. A Lightner, J. Phys. Chem. B 2016.

[37] M. Oda, H. G. Nothofer, U. Scherf, V. Šunjić, D. Richter, W. Regenstein, D. Neher, Macromolecules 2002, 35, 6792-6798

[38] F. C. Spano, S. C. J. Meskers, E. Hennebicq, D. Beljonne, J. Chem. Phys. 2008, 129, 024704

[39] R. Tempelaar, A. Stradomska, J. Knoester, F. C. Spano, J. Phys. Chem B 2011, 115, 10592-10603.

[40] A. B. Nepomnyashchii, M. Bröring, J. Ahrens, A. J. Bard, J. Am. Chem. Soc. 2011, 133, 19498-19504.

[41] M. Bröring, R. Krüger, S. Link, C. Kleeberg, S. Köhler, X. Xie, B Ventura, L. Flamigni, Chem. Eur. J. 2008, 14, 2976-2983.

[42] B. Ventura, G. Marconi, M. Broring, R. Kruger, L. Flamigni, New J. Chem. 2009, 33, 428-438.

[43] X. Ma, E. A. Azeem, X. Liu, Y. Cheng, C. Zhu, J. Mater. Chem. 2014, 2, 1076-1084.

[44] P. H. Schippers, J. P. M. Van der Ploeg, H. P. J. M. Dekkers, J. Am Chem. Soc. 1983, 105, 84-89.

[45] S. Chibani, A. D. Laurent, B. Le Guennic, D. Jacquemin, J. Chem. Theory Comput. 2014, 10, 4574-4582.

[46] S. Chibani, B. Le Guennic, A. Charaf-Eddin, A. D. Laurent, D. Jacquemin, Chem. Sci. 2013, 4, 1950-1963.

[47] S. Chibani, B. Le Guennic, A. Charaf-Eddin, O. Maury, C. Andraud, D. Jacquemin, J. Chem. Theory Comput. 2012, 8, 3303-3313.

[48] Y. Zhao, D. G. Truhlar, Theor. Chem. Acc. 2007, 120, 215-241.

[49] T. D. Crawford, W. D. Allen, Mol. Phys. 2009, 107, 1041 - 1057

[50] B. C. Mort, J. Autschbach, J. Phys. Chem. A 2005, 109, 8617-8623.

[51] M. Pecul, K. Ruud, Phys. Chem. Chem. Phys. 2011, 13, 643-650.

[52] S. Abbate, G. Longhi, F. Lebon, E. Castiglioni, S. Superchi, L. Pisani, F. Fontana, F. Torricelli, T. Caronna, C. Villani, et al., J. Phys. Chem. C 2014, 118, 1682-1695
[53] G. Longhi, E. Castiglioni, S. Abbate, F. Lebon, D. A. Lightner, Chirality 2013, 25, 589-599.

[54] G. Longhi, E. Castiglioni, C. Villani, R. Sabia, S. Menichetti, C. Viglianisi, F. Devlin, S. Abbate, J. Photochem. Photobiol. A 2016, in press. DOI 10.1016/j.jphotochem.2015.1012.1011

[55] G. Longhi, S. Abbate, G. Mazzeo, E. Castiglioni, P. Mussini, T. Benincori, R. Martinazzo, F. Sannicolò, J. Phys. Chem. C 2014, 118, 16019-16027.

[56] R. Fukuda, M. Ehara, Phys. Chem. Chem. Phys. 2013, 15, 1742617434.

[57] D. Padula, D. Picconi, A. Lami, G. Pescitelli, F. Santoro, J. Phys. Chem A 2013, 117, 3355-3368

[58] G. Pescitelli, T. Bruhn, Chirality 2016, 28, 466-474

[59] A. V. Marenich, C. J. Cramer, D. G. Truhlar, C. A. Guido, B. Mennucci, G. Scalmani, M. J. Frisch, Chem. Sci. 2011, 2, 2143-2161.

[60] H. R. McAlexander, T. D. Crawford, J. Chem. Phys. 2015, 142, 154101

[61] A. Schaumlöffel, M. Groh, M. Knauer, A. Speicher, G. Bringmann, Eur. J. Org. Chem. 2012, 2012, 6878-6887.

[62] D. C. G. Götz, A. C. Gehrold, S. J. Dorazio, P. Daddario, L. Samankumara, G. Bringmann, C. Brückner, T. Bruhn, Eur. J. Org. Chem. 2015, 2015, 3913-3922.

[63] Y. Zhao, D. G. Truhlar, J. Phys. Chem. A 2006, 110, 13126-13130.

[64] V. P. Nicu, E. J. Baerends, Phys. Chem. Chem. Phys. 2011, 13, 1612616129.

[65] G. Longhi, M. Tommasini, S. Abbate, P. L. Polavarapu, Chem. Phys. Lett. 2015, 639, 320-325.

[66] L. D. Barron, Molecular Light Scattering and Optical Acticity, Cambridge Univeristy press, Cambridge, 2004

[67] EXAT (Excitonic Analysis Tool). S. Jurinovich, C. A. Guido, B. Mennucci. University of Pisa, Italy, 2014, https://www1.dcci.unipi.it/molecolab/tools/.

[68] T. Kinuta, N. Tajima, M. Fujiki, M. Miyazawa, Y. Imai, Tetrahedron 2012 $68,4791-4796$.

[69] Y. Kitayama, K. Nakabayashi, T. Wakabayashi, N. Tajima, M. Fujiki, Y. Imai, RSC Adv. 2015, 5, 410-415.

[70] V. Barone, A. Baiardi, J. Bloino, Chirality 2014, 26, 588-600.

[71] B. Pritchard, J. Autschbach, ChemPhysChem 2010, 11, 2409-2415

[72] Y. Liu, J. Cerezo, G. Mazzeo, N. Lin, X. Zhao, G. Longhi, S. Abbate, F. Santoro, J. Chem. Theory Comput. 2016, in press. DOI 10.1021/acs.jctc. 1026b00109.

[73] E. Castiglioni, S. Abbate, G. Longhi, Appl. Spectrosc. 2010, 64, 1416 1419

[74] Gaussian 09, Revision D.01. M. J. Frisch, G. W. Trucks, H. B. Schlegel, G. E. Scuseria, M. A. Robb, J. R. Cheeseman, G. Scalmani, V. Barone B. Mennucci, G. A. Petersson, et al. Wallingford CT, 2013

[75] F. Weigend, R. Ahlrichs, Phys. Chem. Chem. Phys. 2005, 7, 3297-3305.

[76] H. Christof, F. Weigend, J. Chem. Phys. 2000, 113, 5154-5161.

[77] A. Hellweg, S. A. Grun, C. Hattig, Phys. Chem. Chem. Phys. 2008, 10, 4119-4127.

[78] F. Furche, R. Ahlrichs, C. Hättig, W. Klopper, M. Sierka, F. Weigend, WIREs Comput. Mol. Sci. 2014, 4, 91-100.

[79] TURBOMOLE, Version 6.6. R. Ahlrichs, M. K. Armbruster, R. A Bachorz, M. Bär, H.-P. Baron, R. Bauernschmitt, F. A. Bischoff, S. Böcker, N. Crawford, P. Deglmann, et al. Karlsruhe, Germany, 2014. 


\section{Entry for the Table of Contents}

\section{FULL PAPER}

Fluorescence and CPL spectra of BODIPY DYEmers $\mathbf{1}$ and $\mathbf{2}$ were measured with our new home-built CPL instrument. 1 has strong ECD and CPL spectra $\left(g_{\text {lum }}=4 \cdot 10^{-3}\right)$ well reproduced by M06-2X and SCS-CC2 calculations. 2 has weaker ECD and CPL spectra $\left(g_{\text {lum }}=4 \cdot 10^{-4}\right)$, due to the mutual cancellation of $\mu-\mu$ and $\mathrm{m}-\mu$ exciton couplings, and to its conformational freedom. The correct $\mathrm{CPL}$ sign could be obtained only with SCS-CC2 or M06-HF calculations.

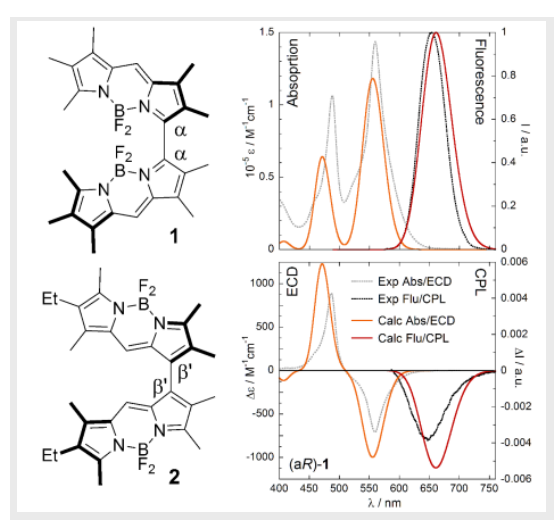

Francesco Zinna, Torsten Bruhn, Ciro A. Guido, Johannes Ahrens, Martin Bröring, Lorenzo Di Bari, Gennaro Pescitelli

Page No. - Page No.

Circularly Polarized Luminescence from Axially Chiral BODIPY DYEmers: an Experimental and Computational Study 